\title{
One-Step Simultaneous Synthesis of Circularly Polarized Luminescent Multiple Helicenes Using a Chrysene Framework
}

\author{
[a] Dr. T. Ikai, S. Yamakawa, N. Suzuki, Prof. E. Yashima \\ Department of Molecular and Macromolecular Chemistry \\ Graduate School of Engineering, Nagoya University \\ Chikusa-ku, Nagoya 464-8603 (Japan) \\ E-mail: ikai@chembio.nagoya-u.ac.jp; yashima@chembio.nagoya-u.ac.jp
}

Tomoyuki Ikai, ${ }^{*[a]}$ Shoya Yamakawa, ${ }^{[a]}$ Nozomu Suzuki, ${ }^{[a]}$ and Eiji Yashima ${ }^{*[a]}$

\begin{abstract}
A series of multiple helicenes was simultaneously synthesized in one step by intramolecular cyclization of a single chrysene derivative containing two 2-[(4-alkoxyphenyl)ethynyl]phenyl units accompanied by rearrangements of the aryl pendants. The electrophile-induced double cyclization with or without aryl migrations proceeded efficiently under acidic conditions to afford annulative $\pi$ extension of the chrysene units and produced quadruple (QH-2), triple (TH-2), and double (DH-2) helicenes containing [4]- and/or [5]helicene frameworks with dynamic and/or static helicene chirality in one step. Three multiple helicenes' structures were determined by $X$-ray crystallography and/or density functional theory calculations. The multiple $\mathrm{TH}-2$ and $\mathrm{DH}-2$ helicenes were separated into enantiomers because of the stable one and two [5]helicene moieties, respectively, and showed intense circular dichroism and circularly polarized luminescence. Although QH-2, which comprises four [4]helicene subunits, was not resolved into enantiomers, the $\mathrm{TH}-2$ enantiomers were further separated into a pair of diastereomers at low temperature resulting from their substituted [4] helicene chirality.
\end{abstract}

\section{Introduction}

Helicenes are unique spiral-shaped polycyclic arenes consisting of more than four consecutive ortho-fused aromatic rings, and they are inherently chiral due to their helicity. ${ }^{[1]}$ Such helically twisted $\pi$-conjugated frameworks with nonplanar geometries have inspired chemists to develop helicene-based functional molecules and polymers for applications in optoelectronic devices ${ }^{[1, i]}$ and as functional materials for chiral sensors and switches, ${ }^{[2]}$ asymmetric catalysis, ${ }^{[3]}$ and circularly polarized luminescence $(\mathrm{CPL}){ }^{[4]}$ Furthermore, $\pi$-extended multiple helicenes comprising two or more helicene subunits fused together have attracted considerable research interest because of their unique multiple static and/or dynamic helical substructures, which can interconvert in a correlated manner, resulting in specific chiral and physical properties different to those of the single helicenes. ${ }^{[1 a, 5]}$ To date, versatile methods to produce multiple helicenes have been reported, ${ }^{[5,6]}$ including oxidative photocyclodehydrogenation and Scholl reactions. However, these methods involve multistep processes, causing low overall yields. Therefore, the chiroptical studies of opticallyactive multiple helicenes and their applications as chiral materials are limited when compared to those of optically-active single helicenes.
Swager et al. reported a facile synthetic approach to polycyclic aromatics and $\pi$-conjugated ladder polymers through acid-catalyzed electrophilic intramolecular multicyclizations (alkyne benzannulations), ${ }^{[7]}$ which has now been expanded to provide a rich variety of ladder-type molecules and polymers. ${ }^{[8]}$ Taking advantage of this acid-catalyzed annulative $\pi$-extension approach combined with the use of a chiral triptycene framework, we have recently succeeded in the synthesis of single-handed helical ladder polymers without any structural defects. ${ }^{[9]}$

Herein we report an unprecedented acid-catalyzed onestep/simultaneous/quantitative synthesis of a series of multiple helicenes, that is, quadruple $(\mathrm{QH}-2)$, triple $(\mathrm{TH}-2)$, and double (DH-2) helicenes, comprising [4]helicene and/or [5]helicene substructures, using a chrysene unit as a key building block. ${ }^{[10]}$ The reaction proceeds through an electrophile-induced double cyclization accompanied by aryl rearrangements with or without aryl migrations (Figure $1 \mathrm{~A}$ ). The mechanism of this reaction, the structures and stereochemical dynamics of the novel multiple helicenes, and the chiroptical properties of the optically-pure TH2 and DH-2 separated by chiral HPLC are described.

\section{Results and Discussion}

One-Step Simultaneous Synthesis of Three Multiple Helicenes Using a Chrysene Framework and Structure Determination

The chrysene derivative $1 \mathrm{a}^{[11]}$ containing two 2-[(4alkoxyphenyl)ethynyl]phenyl units was designed and synthesized (Scheme S1) and treated with trifluoroacetic acid (TFA) in dichloromethane at $25{ }^{\circ} \mathrm{C}$ for $12 \mathrm{~h}$ to obtain the quadruple [4]helicene (QH-2a) via electrophile-induced intramolecular double cyclizations that are reported to occur only at the 5- and 11-positions of the chrysene unit (Figure 1A). ${ }^{[\mathrm{a}, 9]}$ Surprisingly, a triple helicene ( $\mathrm{TH}-2)$ comprising one [5]- and two [4] helicenes fused together as well as a double [5] helicene ( $\mathrm{DH}-2)$ were unexpectedly obtained along with the anticipated quadruple $\mathrm{QH}$ 2a.

In fact, the ${ }^{1} \mathrm{H}$ NMR spectrum of the reaction products obtained after the complete consumption of the two ethynyl groups in 1a within $12 \mathrm{~h}$ (Figure S2A,B; 99\% yield after silica gel chromatography) is complicated (Figure S3A), which implies the formation of an unexpected product mixture. The reaction 
A

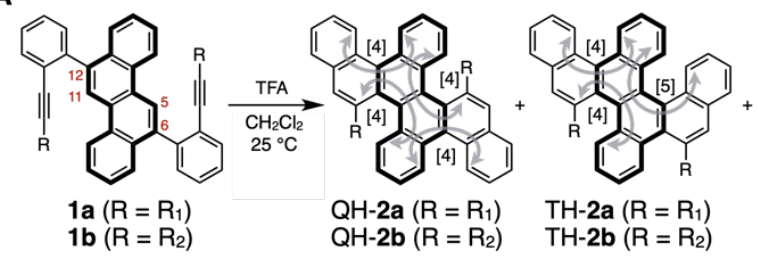

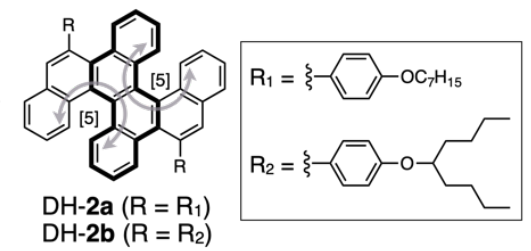

B

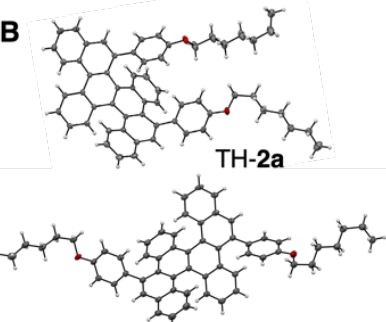

$\mathrm{DH}-2 \mathrm{a}$
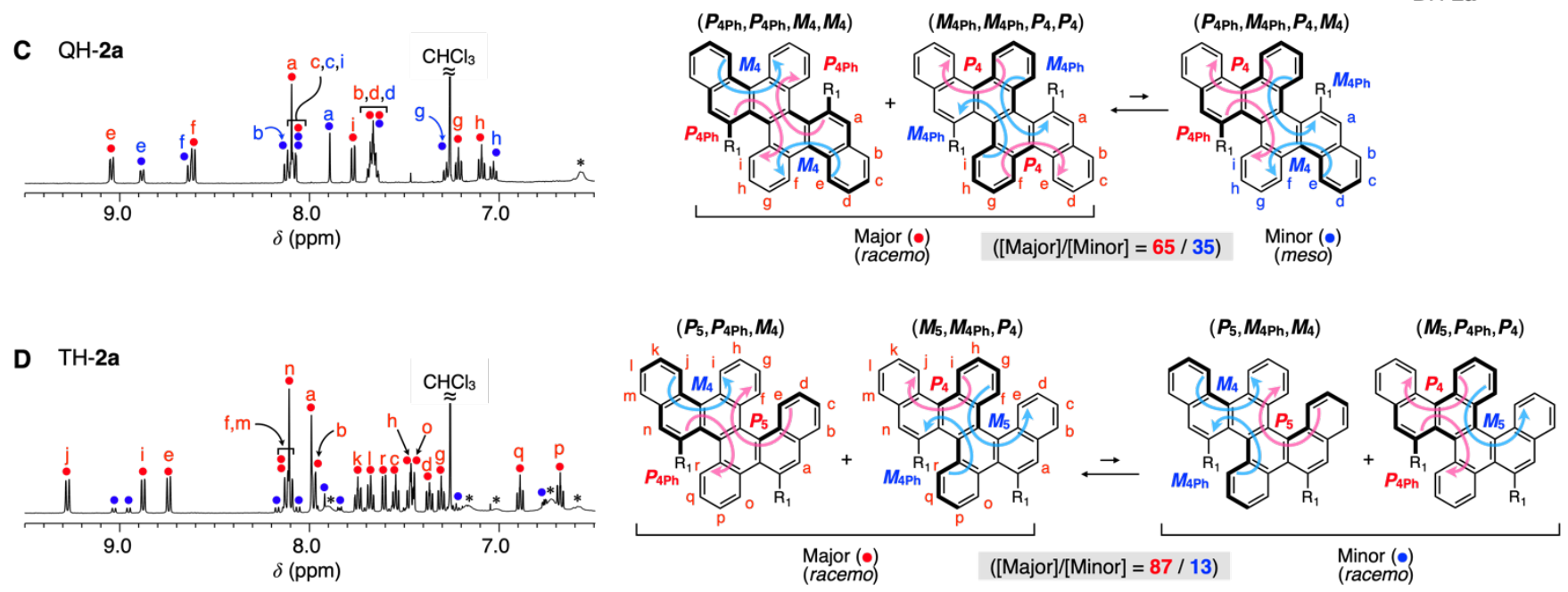

E $\mathrm{DH}-\mathbf{2 a}$
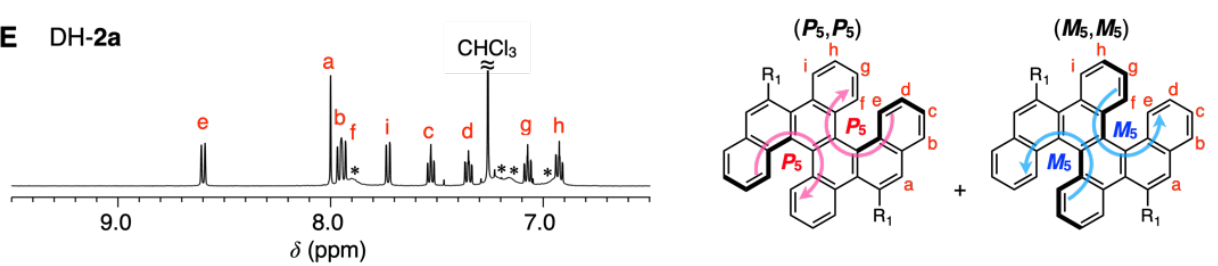

$\mathbf{F}$

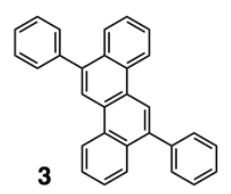

Figure 1. (A) Acid-catalyzed simultaneous synthesis of multiple helicenes (QH-2, TH-2, and DH-2) from a chrysene derivative 1 in dichloromethane/TFA (98/2, $\mathrm{v} / \mathrm{v})$. (B) X-ray crystal structures of $\mathrm{TH}-2 \mathrm{a}$ and $\mathrm{DH}-2 \mathrm{a}$. Thermal ellipsoids are represented at the $50 \%$ probability level. (C-E) ${ }^{1} \mathrm{H} \mathrm{NMR}$ spectra $(500 \mathrm{MHz}, \mathrm{CDCl} 3$, $25^{\circ} \mathrm{C}$ ) of $\mathrm{QH}-2 \mathrm{a}(\mathrm{C}), \mathrm{TH}-2 \mathrm{a}(\mathrm{D})$, and $\mathrm{DH}-2 \mathrm{a}(\mathrm{E})$. For the signal assignments, see Figures S5-S7. Ratios of major and minor components contained in QH-2a and TH-2a estimated by their integral ratios were in good agreement with those calculated by DFT method (see Figure S4 and Tables S3 and S4). Asterisks denote the aromatic protons from alkoxyphenyl pendants $\left(R_{1}\right)$. $(F)$ Structure of 3.

products were separated into just three components by HPLC using chiral columns. Among them, two fractions isolated were unambiguously identified as the triple ( $\mathrm{TH}-2 \mathrm{a})$ and double $(\mathrm{DH}-$ 2a) helicenes, respectively, by single-crystal $X$-ray crystallography, as shown in Figure $1 \mathrm{~B} \cdot{ }^{[12]}$ The simple ${ }^{1} \mathrm{H}$ NMR spectrum of DH-2a (Figure 1E) indicates the presence of only one isomer, a racemic mixture of $\left(P_{5}, P_{5}\right)$ - and $\left(M_{5}, M_{5}\right)-\mathrm{DH}-2 \mathbf{a}$, as supported by chiral separation results (see Figure 3 ), while that of $\mathrm{TH}-2 \mathrm{a}$ indicates a mixture of two diastereomers at a ratio of $87: 13$ (Figure 1D) (for their assignments, see below). Single crystals suitable for X-ray analysis for the third fraction could not be obtained, but its structure was determined to be that of the quadruple [4] helicene (QH-2a) by 2D NMR analyses combined with density functional theory (DFT) calculations (Figures S4A and S5 and Table S3). In solution, QH-2a exists as a mixture of two diastereomers at a ratio of 65:35 (Figure 1C), assigned to the racemo- and meso-diastereomers, respectively, based on DFT calculations (see Figure S4A and Table S3). Thus, based on the ${ }^{1} \mathrm{H}$ NMR spectra of the three multiple helicenes isolated (Figure $1 \mathrm{C}-\mathrm{E})$, the acid-catalyzed electrophile-induced cyclizations of $1 \mathrm{a}$ was found to simultaneously produce $\mathrm{QH}-\mathbf{2 a}, \mathrm{TH}-\mathbf{2 a}$, and $\mathrm{DH}-\mathbf{2 a}$ at a ratio of 26:61:13 with no other byproducts in one step (Figure S3A).

\section{Mechanism of Simultaneous Synthesis of Multiple Helicenes through Acid-Catalyzed Alkyne Benzannulations of 1}

Polycyclic aromatic compounds bearing aryl pendants can potentially undergo aryl migrations under strong acidic conditions, giving a mixture of structural isomers with the aryl groups attached at different positions. ${ }^{[13]}$ Therefore, the unexpectedly formed one (TH-2a) and two (DH-2a) [5]helicene frameworks are most likely due to intramolecular rearrangement along with aryl migrations of one or both of the 2-[(4-alkoxyphenyl)ethynyl]phenyl units from the 6- or 6,12-positions to the 5- or 5,11-positions of the chrysene unit, respectively, followed by an intramolecular cyclization (Figure 1A). However, this type of rearrangement did not occur at all when the analogous chrysene derivative 3 (Figure 1F) was treated with TFA in dichloromethane (Figure S8), which indicates that the phenylethynyl groups of $1 \mathbf{a}$ are indispensable for the observed acid-promoted intramolecular rearrangement/aryl migration on the chrysene unit.

We then propose a plausible mechanism for the formations of [4]- and/or [5] helicene frameworks during the annulative $\pi$ extension of $1 \mathrm{a}$ under acidic conditions, thereby enabling the one- 

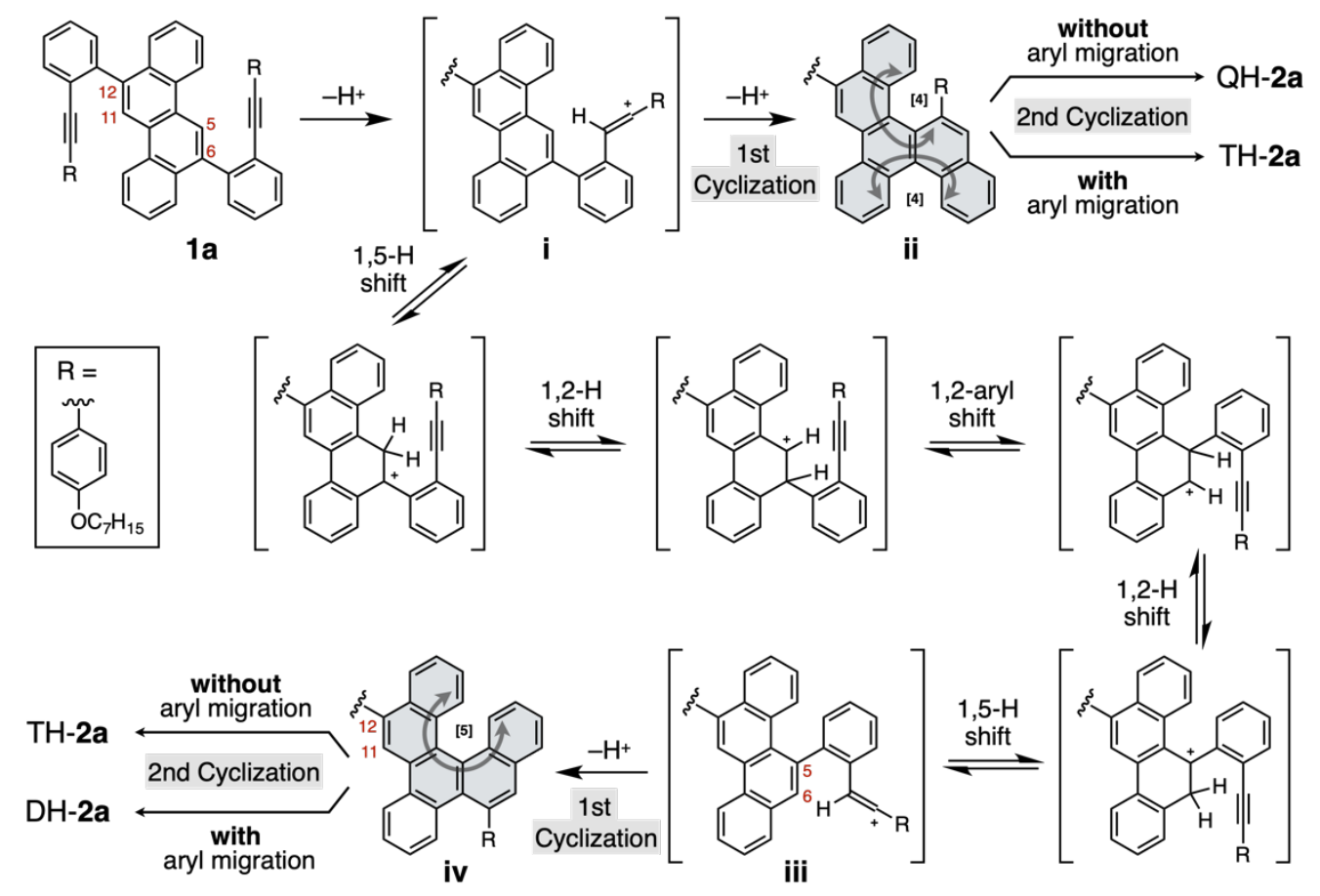

Figure 2. Plausible mechanism for the formation of double [4] helicene (ii) and [5]helicene (iv) frameworks from the corresponding vinyl carbocation intermediates (i and iii, respectively) during the annulative m-extension of a chrysene moiety (1a) with phenylethynyl pendants under acidic conditions.

step simultaneous synthesis of a series of multiple helicenes (Figure 2). Briefly, the vinyl carbocation intermediate $\mathbf{i}$ formed from 1a through protonation of the ethynyl groups ${ }^{[7]}$ converts into the expected double [4] helicene framework ii by a subsequent electrophile-induced intramolecular cyclization. Instead of this cyclization reaction, the vinyl carbocation $\mathbf{i}$ undergoes hydride and aryl shifts to produce another vinyl carbocation intermediate iii. Subsequent intramolecular cyclization results in the formation of the [5]helicene framework iv. In the same manner, a second intramolecular cyclization with or without aryl migrations also proceeds on another side of the chrysene unit, thus simultaneously yielding $\mathrm{QH}-\mathbf{2 a}, \mathrm{TH}-\mathbf{2 a}$, and $\mathrm{DH}-2 \mathbf{a}$ containing [4] helicene and/or [5]helicene frameworks in one step. To the best of our knowledge, this is the first example of a one-step simultaneous synthesis of three kinds of discrete multiple helicenes comprising different numbers of helicene moieties from a single chrysene unit through acid-catalyzed intramolecular cyclizations with or without a combination of rearrangements of aryl pendants that readily proceeds quantitatively. Bock, Durola, and coworkers reported a unique $\mathrm{Pd}$-catalyzed cyclization reaction of a chrysene derivative in which a double cyclization and rearrangement occurs concurrently, affording a double helicene together with presumably two kinds of multiple helicenes in relatively low yields. However, the products were not fully isolated; therefore, their exact structures and chiroptical properties remained unknown. ${ }^{[10 a]}$

Resolution of Multiple Helicenes into Enantiomers and/or Diastereomers, Their Chiroptical Properties, and Stepwise Helicity Inversion

We then investigated whether the chrysene-based multiple helicenes could be separated into enantiomers and/or diastereomers by chiral HPLC. To make preparative-scale resolutions by chiral HPLC easier, analogous multiple helicenes carrying branched alkoxy groups, QH-2b, TH-2b, and $\mathrm{DH}-\mathbf{2} \mathbf{b}$, showing better solubilities than the corresponding 2a-type helicenes, were prepared from $\mathbf{1 b}$ in the same way (Figures $1 \mathrm{~A}$, S3B, and S9-S12). As anticipated, the double helicene DH-2b was completely separated into two fractions at $0{ }^{\circ} \mathrm{C}$ (Figure $3 \mathrm{~A}-$ $\mathrm{C})$ and the isolated products presented mirror-image $\mathrm{CD}$ spectra and identical absorption spectra (Figure 3D). This result indicates that they are the enantiomeric pair $\left(P_{5}, P_{5}\right)-$ and $\left(M_{5}, M_{5}\right)-\mathrm{DH}-\mathbf{2 b}$ and that the corresponding achiral meso isomer, $\left(P_{5}, M_{5}\right)-\mathrm{DH}-2 \mathbf{b}$, is not produced, as supported by its simple ${ }^{1} \mathrm{H}$ NMR spectrum (Figure S9C), which, like that of DH-2a, shows one set of signals derived from rac-DH-2b. The absolute configurations (helicities) of the first- and second-eluted enantiomers of $\mathrm{DH}-\mathbf{2 b}$ were assigned as $\left(M_{5}, M_{5}\right)$ and $\left(P_{5}, P_{5}\right)$, respectively, based on timedependent-DFT calculations (Figure S13).
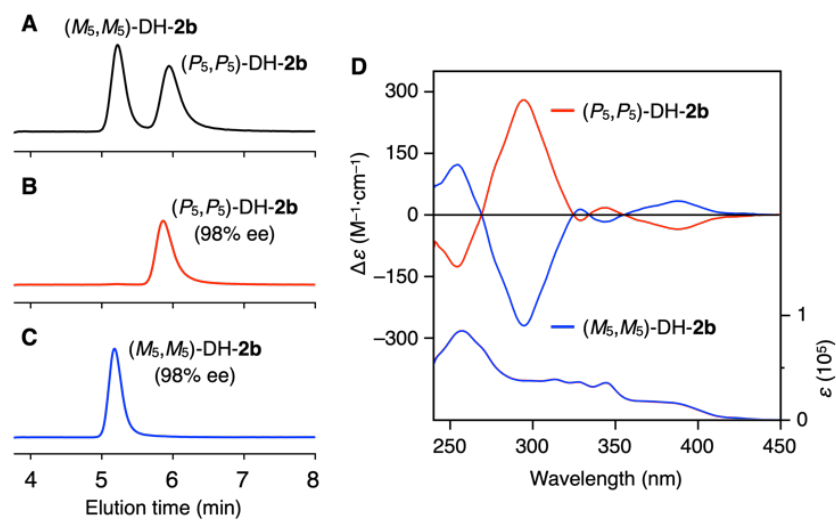

Figure 3. (A-C) Chromatograms for the resolution of rac-DH-2b $(\mathrm{A})$ and the fractionated $\left(P_{5}, P_{5}\right)-\mathrm{DH}-2 \mathbf{b}(\mathrm{B})$ and $\left(M_{5}, M_{5}\right)-\mathrm{DH}-2 \mathbf{b}(\mathrm{C})$. Chromatographic conditions: column, CHIRALPAK IA $(0.46 \mathrm{~cm}$ (i.d.) $\times 25 \mathrm{~cm})$; eluent, $n$ hexane/chloroform $(95 / 5, \mathrm{v} / \mathrm{v})$; flow rate, $1.0 \mathrm{~mL} / \mathrm{min}$; temperature, $0{ }^{\circ} \mathrm{C}$. (D) $\mathrm{CD}$ and absorption spectra of $\left(P_{5}, P_{5}\right)-\mathrm{DH}-\mathbf{2 b}(0.15 \mathrm{mM})$ and $\left(M_{5}, M_{5}\right)-\mathrm{DH}-\mathbf{2 b}$ $(0.13 \mathrm{mM})$ in chloroform at $25^{\circ} \mathrm{C}$. The absolute configurations were assigned 3 based on the time-dependent-DFT calculations (Figure S13). 

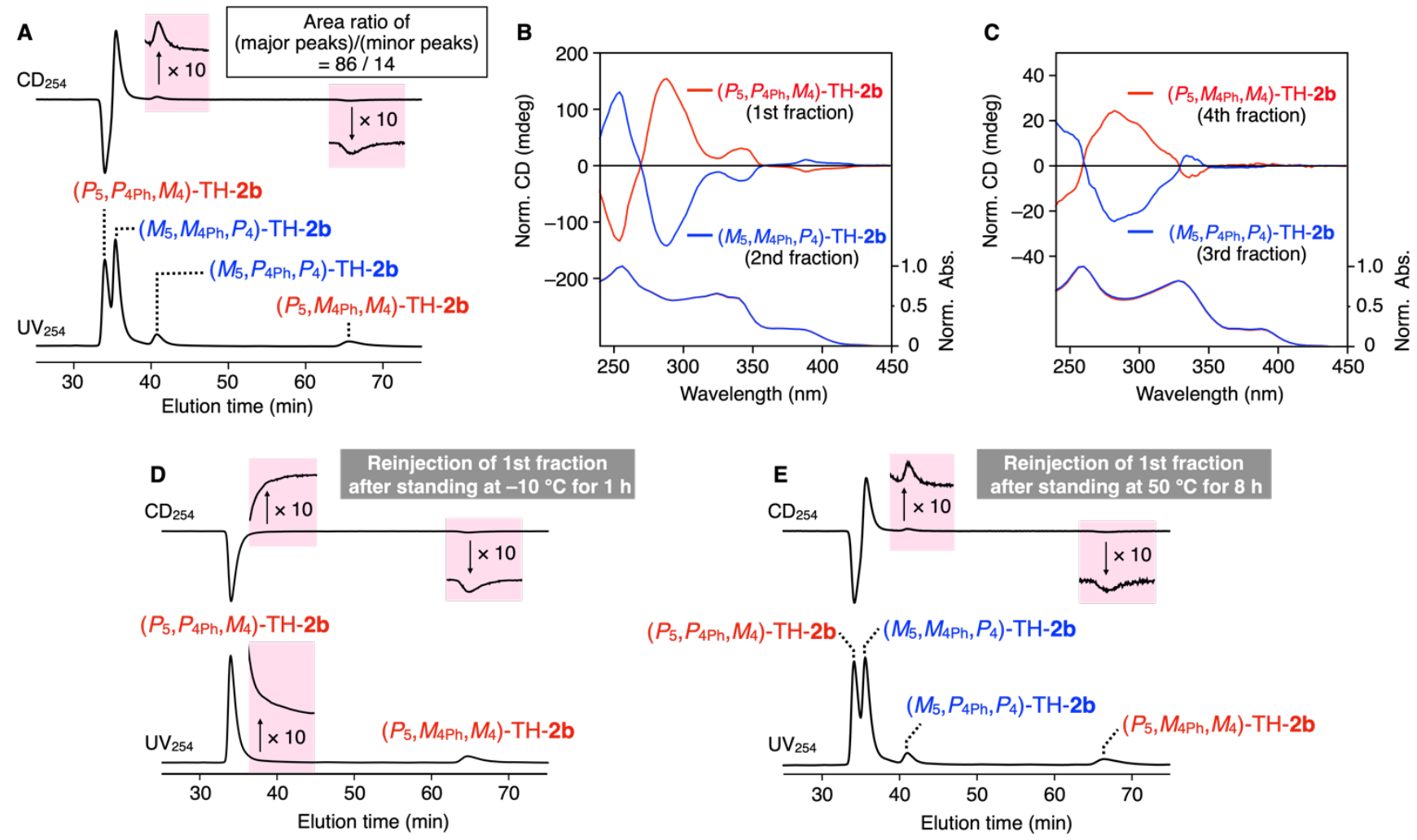

Figure 4. (A) Chromatogram for the resolution of TH-2b with dual UV and CD detectors. Chromatographic conditions: columns, CHIRALPAK IA-3 (0.46 cm (i.d.) $\times 15 \mathrm{~cm})$, CHIRALPAK IB-3 $(0.46 \mathrm{~cm}$ (i.d.) $\times 15 \mathrm{~cm})$, and CHIRALPAK IA $(0.46 \mathrm{~cm}$ (i.d.) $\times 25 \mathrm{~cm})$ were connected in series; eluent, $n$ hexane/dichloromethane $(91 / 9, \mathrm{v} / \mathrm{v})$; flow rate, $0.50 \mathrm{~mL} \mathrm{~min}^{-1}$; temperature, $-20{ }^{\circ} \mathrm{C}$. (B,C) $\mathrm{CD}$ and absorption spectra of the first- $\left(\left(P_{5}, P_{4 \mathrm{Ph}}, M_{4}\right)-\mathrm{TH}-2 \mathrm{~b}\right)$ and second-eluted $\left(\left(M_{5}, M_{4 \mathrm{Ph}}, P_{4}\right)-\mathrm{TH}-2 \mathbf{b}\right)$ components in A $(\mathrm{B})$ and the third- $\left(\left(M_{5}, P_{4 \mathrm{Ph}}, P_{4}\right)-\mathrm{TH}-2 \mathrm{~b}\right)$ and fourth-eluted $\left(\left(P_{5}, M_{4 \mathrm{Ph}}, M_{4}\right)-\mathrm{TH}-2 \mathrm{~b}\right)$ components in A $(\mathrm{C})$ in $n$ hexane/dichloromethane $(91 / 9, \mathrm{v} / \mathrm{v})$ at room temperature, obtained by stop-flow HPLC-CD analysis. The CD and absorption spectra were normalized based on the corresponding absorption spectra at room temperature. (D,E) HPLC chromatograms of the fractionated first-eluted component in A $\left(\left(P_{5}, P_{4 \mathrm{Ph}}, M_{4}\right)-\mathrm{TH}-\right.$ 2b) after standing at $-10^{\circ} \mathrm{C}$ for $1 \mathrm{~h}(\mathrm{D})$ and at $50{ }^{\circ} \mathrm{C}$ for $8 \mathrm{~h}$ with dual UV and CD detectors (E). For chromatographic conditions, see caption (A). The absolute configurations were assigned based on time-dependent DFT calculations (Figure S16).

The CD signals of $\left(M_{5}, M_{5}\right)$-DH-2b gradually decrease with time in toluene at high temperatures $\left(40-80^{\circ} \mathrm{C}\right)$ (Figure S14A), which suggests that the racemization is a result of the inversion of the [5]helicene substructures, as often seen in single [5] helicenes that are non-substituted in the fjord region. ${ }^{[1 d, 14]}$ The racemization process obeys the first-order kinetics in the absence of achiral meso- $\left(P_{5}, M_{5}\right)-\mathrm{DH}-\mathbf{2} \mathbf{b}$ formation as supported by the time-dependent enantioseparation and $C D$ intensity change results (Figure S15). The activation energy $\left(E_{\mathrm{a}}\right)$ for the racemization of $\mathrm{DH}-\mathbf{2} \mathbf{b}$ in toluene was then estimated to be 26.1 kcal $\mathrm{mol}^{-1}$ (Figure S14), which is higher than that of [5]helicene $\left(E_{\mathrm{a}}=23.5 \mathrm{kcal} \mathrm{mol}^{-1}\right){ }^{[14]}$ These results also indicate that the dynamic helicities of the two [5] helicene units fused together in $\mathrm{DH}-\mathbf{2} \mathbf{b}$ interconvert in a correlated manner with each other.

The triple helicene (TH-2b), which comprises two [4]- and one [5]helicene substructures, was resolved into two pairs of major and minor peaks by chiral HPLC at $-20^{\circ} \mathrm{C}$ (Figure 4A), where each pair showed the mirror-imaged $C D$ spectra (Figure $4 B, C)$. DFT calculations for all four possible diastereomers of a model TH-2 with the same $(P)-[5]$ helicene subunit (Figure S4B and Table S4) revealed that one pair of major peaks can be assigned to the most stable $\left(P_{5}, P_{4 \mathrm{Ph}}, M_{4}\right.$ and $\left.M_{5}, M_{4 \mathrm{Ph}}, P_{4}\right)-\mathrm{TH}-2 \mathbf{b}$, whose structures are identical to those determined by $\mathrm{X}$-ray crystallography (Figure 1B). Accordingly, one pair of minor peaks is assigned to the next most stable $\left(P_{5}, M_{4 \mathrm{Ph}}, M_{4}\right.$ and $\left.M_{5}, P_{4 \mathrm{Ph}}, P_{4}\right)-$ $\mathrm{TH}-\mathbf{2 b}$. The calculated relative populations (85.5:14.4) are in good agreement with the major and minor peak ratios observed in the
${ }^{1} \mathrm{H}$ NMR spectrum of TH-2b (87:13 in Figure S9B) and the chromatographic separation result for TH-2b (86:14 in Figure 4A). Based on comparison between the experimental and calculated CD spectra, the absolute configurations of the first-, second-, third-, and fourth-eluted stereoisomers are assigned as $\left(P_{5}, P_{4 \mathrm{Ph}}, M_{4}\right), \quad\left(M_{5}, M_{4 \mathrm{Ph}}, P_{4}\right), \quad\left(M_{5}, P_{4 \mathrm{Ph}}, P_{4}\right), \quad$ and $\quad\left(P_{5}, M_{4 \mathrm{Ph}}, M_{4}\right)$, respectively (Figure $\mathrm{S} 16)$.

Interestingly, when the first-eluted stereoisomer, $\left(P_{5}, P_{4 \mathrm{Ph}}, M_{4}\right)-\mathrm{TH}-2 \mathrm{~b}$, fractionated by chiral HPLC at $-20^{\circ} \mathrm{C}$, was reinjected into the chiral HPLC after storage at $-10^{\circ} \mathrm{C}$ for $1 \mathrm{~h}$, an additional minor peak $\left(\left(P_{5}, M_{4 \mathrm{Ph}}, M_{4}\right)-\mathrm{TH}-2 \mathrm{~b}\right)$ converted from the $\left(P_{5}, P_{4 \mathrm{Ph}}, M_{4}\right)-\mathrm{TH}-2 \mathrm{~b}$ appeared in the chromatogram (Figure 4D); its diastereomeric ratio was almost the same as that in the original chromatogram (Figure 4A). Hence, only the inversion of the phenyl-substituted [4]helicene unit in TH-2b $\left(P_{4 \mathrm{Ph}}\right.$ and $\left.M_{4 \mathrm{Ph}}\right)$ took place at $-10{ }^{\circ} \mathrm{C}$, while maintaining the $P_{5}$ and $M_{4}$ helicities that appear to interconvert in a correlated way, because the $\left(P_{5}, P_{4 \mathrm{Ph}}, P_{4}\right)-\quad$ and $\left(P_{5}, M_{4 \mathrm{Ph}}, P_{4}\right)-\mathrm{TH}-2 \mathrm{~b}$ diastereomers are energetically very unstable (Figure $\mathrm{S}_{4}$ ). The fractionated firsteluted $\left(P_{5}, P_{4 \mathrm{Ph}}, M_{4}\right)-\mathrm{TH}-2 \mathbf{b}$ racemized to a substantial extent over $8 \mathrm{~h}$ at $50^{\circ} \mathrm{C}$ (Figure 4E). These chiral HPLC results clearly reveal the stepwise inversion of helicities in the triple $\mathrm{TH}-\mathbf{2 b}$ helicene in a sequence such that inversion of the phenyl-substituted [4] helicene first takes place, followed by inversion of the [5]helicene that occurs concurrently with inversion of the nonsubstituted [4]helicene in a stereochemically correlated manner. ${ }^{[15]}$ 

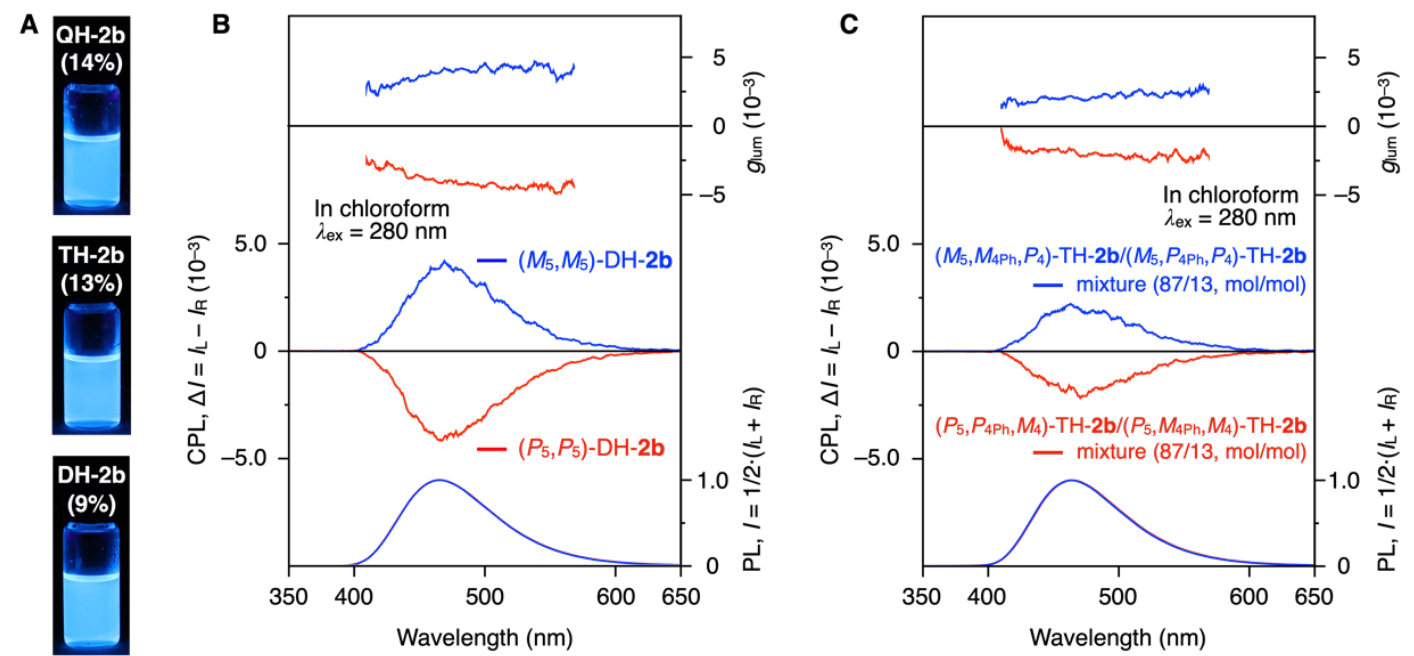

Figure 5. (A) Photographs of $\mathrm{QH}-\mathbf{2 b}$ (top), $\mathrm{TH}-\mathbf{2 b}$ (middle), and $\mathrm{DH}-\mathbf{2 b}$ (bottom) in chloroform under irradiation at $365 \mathrm{~nm}$. [2b] = ca. $0.1 \mathrm{mM}$. Fluorescence quantum yields are shown in the parentheses. (B,C) Normalized PL (bottom), CPL (middle), and glum (top) spectra of $\left(P_{5}, P_{5}\right)$-DH-2b $(0.15 \mathrm{mM})$ and $\left(M_{5}, M_{5}\right)$ $\mathrm{DH}-2 \mathrm{~b}(0.13 \mathrm{mM})$ and a mixture of $\left(P_{5}, P_{4 \mathrm{Ph}}, M_{4}\right)$ - and $\left(P_{5}, M_{4 \mathrm{Ph}}, M_{4}\right)-\mathrm{TH}-2 \mathrm{~b}(87 / 13, \mathrm{~mol} / \mathrm{mol} ;[\mathbf{2 b}]=0.11 \mathrm{mM})$ and a mixture of $\left(M_{5}, M_{4 \mathrm{Ph}}, P_{4}\right)-$ and $\left(M_{5}, P_{4 \mathrm{Ph}}, P_{4}\right)-\mathrm{TH}-$ 2b $(87 / 13, \mathrm{~mol} / \mathrm{mol} ;[\mathbf{2 b}]=0.10 \mathrm{mM})(\mathrm{C})$ in chloroform at $25{ }^{\circ} \mathrm{C}$. The glum values are defined as $2\left(I_{L}-I_{R}\right) /\left(I_{L}+I_{R}\right)$, where $I_{L}$ and $I_{R}$ are the $P L$ intensities of the left- and right-handed circularly polarized light, respectively. $\lambda_{\mathrm{ex}}=280 \mathrm{~nm}$.

Because of the difficulty in handling the enantiomerically and diastereomerically pure forms of $\mathrm{TH}-\mathbf{2 b}$, the subsequent $\mathrm{CPL}$ studies were performed using $\left(P_{5}, M_{4}\right)$ - and $\left(M_{5}, P_{4}\right)-\mathrm{TH}-\mathbf{2 b}$, in which the helicities of the phenyl-substituted [4] helicene units are in dynamic equilibrium. Optically-active $\left(P_{5}, M_{4}\right)$ - and $\left(M_{5}, P_{4}\right)-\mathrm{TH}$ 2b with optical purities of $>98 \%$ ee were obtained by chiral HPLC under modified chromatographic conditions affording better resolution of $\left(P_{5}, P_{4 \mathrm{Ph}}, M_{4}\right)$ - and $\left(M_{5}, M_{4 \mathrm{Ph}}, P_{4}\right)$-isomers (Figure $\mathrm{S} 17$; for racemization kinetics and thermodynamics for $\left(P_{5}, M_{4}\right)-\mathrm{TH}-2 \mathbf{b}$, see Figure $\mathrm{S18}$ ). QH-2b could not be resolved by chiral HPLC under any of the conditions investigated, most likely because of four [4] helicene subunits with a low helicity inversion barrier. ${ }^{[16]}$

\section{Circularly Polarized Luminescence from Optically-Active Double and Triple Helicenes}

Optically-inactive $\mathrm{DH}-\mathbf{2} \mathbf{b}, \mathrm{TH}-\mathbf{2} \mathbf{b}$, and $\mathrm{QH}-\mathbf{2} \mathbf{b}$ showed $\mathbf{a}$ clear blue fluorescence emission in chloroform under UV irradiation $(365 \mathrm{~nm})$ as shown in Figure $5 \mathrm{~A}$, and their quantum yields were determined to be approximately $10 \%$. As expected, the $\mathrm{DH}-\mathbf{2} \mathbf{b}$ and $\mathrm{TH}-\mathbf{2} \mathbf{b}$ enantiomers obtained just after resolution exhibited clear CPL signals with opposite signs in the corresponding fluorescence regions due to the helicities of the multiple helicenes and their maximum luminescence dissymmetry factors $\left(\left|g_{\text {lum }}\right|\right)$ reached $5.0 \times 10^{-3}$ and $2.5 \times 10^{-3}$, respectively (Figure $5 \mathrm{~B}$ and $\mathrm{C}$ ). The resulting $\left|g_{\mathrm{lum}}\right|$ values are sufficiently high comparable to those of previously reported helicene-based $\mathrm{CPL}$ materials (typically in the range $\left.10^{-5}-10^{-3}\right){ }^{[4 b, c, 17]}$

\section{Conclusion}

In summary, we have found the unprecedented one-step simultaneous formations of three multiple helicenes (double, triple, and quadruple helicenes) comprising [4]- and/or [5]helicene subunits using chrysene-based starting materials through electrophile-induced intramolecular cyclizations with or without a combination of rearrangements of aryl pendants. Two of the multiple helicenes were completely separated into enantiomers and/or diastereomers by chiral HPLC. A unique temperaturedriven stepwise helicity inversion of two [4] helicene substructures with dynamic helical chiralities was, for the first time, directly visualized by chiral HPLC analyses of the triple helicene, providing access to new chiral molecular motors. The opticallyactive multiple helicenes emit left- and right-handed circularly polarized light with a $|g| \mathrm{lum} \mid$ value up to $5.0 \times 10^{-3}$. We believe that the present methodology will be applicable to one-step selective synthesis of further complicated multiple helicenes as well as nonplanar $\pi$-conjugated molecules and curved nanographenes in a predictable manner by the rational molecular design of cyclization precursors. Work towards these goals is now underway in our laboratory.

\section{Acknowledgements}

We thank Professor Makoto Yamashita and Dr. Katsunori Suzuki (Nagoya University) for X-ray crystallographic analysis of TH-2a and DH-2a and Dr. Keiko Kuwata (Nagoya University) for highresolution mass spectrometric analysis. This work was supported by JSPS KAKENHI (Grant-in-Aid for Specially Promoted Research, No. $18 \mathrm{H} 05209$ (E.Y. and T.I.).

\section{Conflict of interest}

The authors declare no conflict of interest.

Keywords: chirality $\bullet$ circularly polarized luminescence $\cdot$ multiple helicenes $\cdot$ alkyne benzannulations $\bullet$ stepwise helicity inversion

a) R. H. Martin, Angew. Chem., Int. Ed. Engl. 1974, 13, 649-660; b) Y. Shen, C.-F. Chen, Chem. Rev. 2012, 112, 1463-1535; c) M. Gingras, Chem. Soc. Rev. 2013, 42, 968-1006; d) M. Gingras, G. Felix, R. Peresutti, Chem. Soc. Rev. 2013, 42, 1007-1050; e) M. Gingras, Chem. Soc. Rev. 2013, 42, 1051-1095; f) J. Bosson, J. Gouin, J. Lacour, 
Chem. Soc. Rev. 2014, 43, 2824-2840; g) I. Stary, I. G. Stara, Targets Heterocycl. Syst. 2017, 21, 23-53; h) M Hasan, V. Borovkov, Symmetry 2018, 10, 10; i) K. Dhbaibi, L. Favereau, J. Crassous, Chem. Rev. 2019, 119, 88468953; j) F. Pop, N. Zigon, N. Avarvari, Chem. Rev. 2019 119, 8435-8478; k) I. G. Stara, I. Stary, Acc. Chem. Res. 2020, 53, 144-158.

[2] a) H. Isla, J. Crassous, C. R. Chim. 2016, 19, 39-49; b) M. Shigeno, Y. Kushida, M. Yamaguchi, Chem. Commun. 2016, 52, 4955-4970; c) J. R. Brandt, F. Salerno, M. J. Fuchter, Nat. Rev. Chem. 2017, 1, 0045.

[3] a) M. J. Narcis, N. Takenaka, Eur. J. Org. Chem. 2014, 2014, 21-34; b) C. S. Demmer, A. Voituriez, A. Marinetti, C. R. Chim. 2017, 20, 860-879; c) J. OuYang, J. Crassous, Coord. Chem. Rev. 2018, 376, 533-547.

[4] a) J. E. Field, G. Muller, J. P. Riehl, D. Venkataraman, J. Am. Chem. Soc. 2003, 125, 11808-11809; b) H. Tanaka, Y. Inoue, T. Mori, ChemPhotoChem 2018, 2, 386-402; c) W. L. Zhao, M. Li, H.-Y. Lu, C.-F. Chen, Chem. Commun. 2019, 55, 13793-13803

[5] a) C. Li, Y. Yang, Q. Miao, Chem. - Asian J. 2018, 13, 884894; b) K. Kato, Y. Segawa, K. Itami, Synlett 2019, 30, 370377 ; c) E. S. Gauthier, R. Rodríguez, J. Crassous, Angew. Chem., Int. Ed. 2020, 59, 22840-22856; d) X. Xu, K. Müllen, A. Narita, Bull. Chem. Soc. Jpn. 2020, 93, 490-506; e) T. Hosokawa, A. Tsurusaki, K. Kamikawa, J. Synth. Org. Chem. Jpn. 2020, 78, 1013-1020.

[6] For recent examples of multiple helicenes, see: a) X. Guo, Z. Yuan, Y. Zhu, Z. Li, R. Huang, Z. Xia, W. Zhang, Y. Li, J. Wang, Angew. Chem., Int. Ed. 2019, 58, 16966-16972; b) Y. Hu, G. M. Paterno, X.-Y. Wang, X.-C. Wang, M. Guizzardi, Q. Chen, D. Schollmeyer, X.-Y. Cao, G. Cerullo, F. Scotognella, K. Muellen, A. Narita, J. Am. Chem. Soc. 2019, 141, 12797-12803; c) H.-A. Lin, K. Kato, Y. Segawa, L. T. Scott, K. Itami, Chem. Sci. 2019, 10, 2326-2330; d) G. Liu, T. Koch, Y. Li, N. L. Doltsinis, Z. Wang, Angew. Chem., Int. Ed. 2019, 58, 178-183; e) Y. Wang, Z. Yin, Y. Zhu, J. $\mathrm{Gu}, \mathrm{Y}$. Li, J. Wang, Angew. Chem., Int. Ed. 2019, 58, 587591; f) F. Zhang, E. Michail, F. Saal, P. Ravat, A.-M. Krause, Chem. - Eur. J. 2019, 25, 16241-16245; g) S. H. Pun, Y. Wang, M. Chu, C. K. Chan, Y. Li, Z. Liu, Q. Miao, J. Am. Chem. Soc. 2019, 141, 9680-9686; h) M. Roy, V. Berezhnaia, M. Villa, N. Vanthuyne, M. Giorgi, J.-V. Naubron, S. Poyer, V. Monnier, L. Charles, Y. Carissan, D. Hagebaum-Reignier, J. Rodriguez, M. Gingras, Y. Coquerel, Angew. Chem., Int. Ed. 2020, 59, 3264-3271; i) F. Zhou, F. Zhou, R. Su, Y. Yang, J. You, Chem. Sci. 2020, 11, 7424-7428; j) N. J. Schuster, L. A. Joyce, D. W. Paley, F. Ng, M. L. Steigerwald, C. Nuckolls, J. Am. Chem. Soc. 2020, 142, 7066-7074; k) N. Ogawa, Y. Yamaoka, H. Takikawa, K.-i. Yamada, K. Takasu, J. Am. Chem. Soc. 2020, 142, 13322-13327. I) S. Ma, J. Gu, C. Lin, Z. Luo, Y. Zhu, J. Wang, J. Am. Chem. Soc. 2020, 142, 16887-16893.

[7] a) M. B. Goldfinger, T. M. Swager, J. Am. Chem. Soc. 1994 116, 7895-7896; b) M. B. Goldfinger, K. B. Crawford, T. M. Swager, J. Am. Chem. Soc. 1997, 119, 4578-4593.

[8] a) W. Chalifoux, W. Yang, Synlett 2017, 28, 625-632; b) Y. C. Teo, H. W. H. Lai, Y. Xia, Chem. - Eur. J. 2017, 23, 14101-14112.

[9] T. Ikai, T. Yoshida, K.-i. Shinohara, T. Taniguchi, Y. Wada, T. M. Swager, J. Am. Chem. Soc. 2019, 141, 4696-4703.

[10] Syntheses of multiple helicenes using chrysene derivatives have been reported previously. For leading examples, see: a) H. Bock, S. Huet, P. Dechambenoit, E. A. Hillard, F. Durola, Eur. J. Org. Chem. 2015, 2015, 1033-1039; b) R. K. Mohamed, S. Mondal, J. V. Guerrera, T. M. Eaton, T. E. Albrecht-Schmitt, M. Shatruk, I. V. Alabugin, Angew. Chem. Int. Ed. 2016, 55, 12054-12058; c) M. Ferreira, G. Naulet, H. Gallardo, P. Dechambenoit, H. Bock, F. Durola, Angew. Chem., Int. Ed. 2017, 56, 3379-3382; d) W. Matsuoka, H. Ito, K. Itami, Angew. Chem., Int. Ed. 2017, 56, 12224 12228.

[11] 1a exists as two diastereomers, probably meso- and racemo-isomers with a slow interconversion on the NMR time scale at room temperature. For details, see Figure S1.
[12] Racemic crystals of $\left(P_{5}, P_{4 \mathrm{Ph}}, M_{4}\right.$ and $\left.M_{5}, M_{4 \mathrm{Ph}}, P_{4}\right)-\mathrm{TH}-2 \mathrm{a}$ and $\left(P_{5}, P_{5^{-}}\right.$and $\left.M_{5}, M_{5}\right)-\mathrm{DH}-2 \mathrm{a}$ were obtained by slow evaporation of their solutions in dichloromethane/ $n$-pentane (see Section 4 in the Supporting Information (SI) and Tables S4 and S5). Only the structures of the single enantiomers are shown in Figure 1B. The helicities of the [5]helicene, the phenyl-substituted [4]helicene, and the other [4] helicene are denoted as $\left(P_{5} / M_{5}\right),\left(P_{4 \mathrm{Ph}} / M_{4 \mathrm{Ph}}\right)$, and $\left(P_{4} / M_{4}\right)$, respectively.

[13] a) H. Wynberg, A. P. Wolf, J. Am. Chem. Soc. 1963, 85, 3308-3308; b) A. Necula, A. Racoveanu-Schiketanz, M. D. Gheorghiu, L. T. Scott, J. Org. Chem. 1995, 60, 3448-3451. [14] C. Goedicke, H. Stegemeyer, Tetrahedron Lett. 1970, 11, 937-940.

[15] Stepwise inversion of helicities in multiple helicenes containing [4] helicene substructures was reported using DFT calculations and NMR, but their direct observations have not been reported. See: Y. Yang, L. Yuan, B. Shan, Z. Liu, Q. Miao, Chem. - Eur. J. 2016, 22, 18620-18627.

[16] S. Grimme, S. D. Peyerimhoff, Chem. Phys. Lett. 1996, 204, 411-417.

[17] For leading examples of circularly polarized luminescent multiple helicenes, see: a) K. Nakamura, S. Furumi, M. Takeuchi, T. Shibuya, K. Tanaka, J. Am. Chem. Soc. 2014, 136, 5555-5558; b) M. Satoh, Y. Shibata, K. Tanaka, Chem. - Eur. J. 2018, 24, 5434-5438; c) H. Tanaka, Y. Kato, M. Fujiki, Y. Inoue, T. Mori, J. Phys. Chem. A 2018, 122, 73787384; d) H. Tanaka, M. Ikenosako, Y. Kato, M. Fujiki, Y. Inoue, T. Mori, Commun. Chem. 2018, 1, 38; e) R. Yamano, Y. Shibata, K. Tanaka, Chem. - Eur. J. 2018, 24, 63646370; f) C. M. Cruz, I. R. Márquez, S. Castro-Fernández, J. M. Cuerva, E. Macôas, A. G. Campana, Angew. Chem., Int. Ed. 2019, 58, 8068-8072; g) B. Liu, M. Bockmann, W. Jiang, N. L. Doltsinis, Z. H. Wang, J. Am. Chem. Soc. 2020, 142, 7092-7099; h) Z. Sun, C. Yi, Q. Liang, C. Bingi, W. Zhu, P. Qiang, D. Wu, F. Zhang, Org. Lett. 2020, 22, 209-213; i) A. Yubuta, T. Hosokawa, M. Gon, K. Tanaka, Y. Chujo, A. Tsurusaki, K. Kamikawa, J. Am. Chem. Soc. 2020, 142, 10025-10033. 